\title{
EVALUATION OF EFFECTIVENESS OF PATHOLOGY REPORTS IN ACTIVE SURVEILLANCE OF TUBERCULOSIS
}

\author{
Özlem Terzi', Derya Öztomurcuk², Seda Gün³, Zeki Kılıçaslan ${ }^{4}$ \\ ${ }^{1}$ Department of Public Health, Faculty of Medicine, Ondokuz Mayis University, Samsun, Turkey \\ 2TB Provincial Coordination Office, Samsun, Turkey \\ ${ }^{3}$ Department of Pathology, Faculty of Medicine, Ondokuz Mayis University, Samsun, Turkey \\ ${ }^{4}$ Department of Chest Diseases, Medical Faculty, Istanbul University, Istanbul, Turkey
}

\section{SUMMARY}

Objective: Despite advancing technology, national TB surveillance systems are still inadequate in terms of patient detection around the world. It was aimed to investigate suspicious cases detected by active surveillance method in pathology laboratories and to evaluate the effectiveness of this method in terms of finding new TB cases.

Methods: This is a descriptive cross-sectional study. It was administrated in Samsun, Turkey, between January 2012-December 2017. Within the scope of active surveillance, pathology laboratories were regularly visited and reported cases with granulomatous inflammation were assessed. The obtained patient list was compared with the records of the Electronic Tuberculosis Management System (ETMS). Patients who were not included in these records were invited to the dispensary and evaluated for TB. They were also referred to the relevant hospitals for diagnosis if necessary. Frequency values and descriptive statistics were calculated using SPSS method.

Results: It was found that $35.6 \%$ of 703 patients with the diagnosis of granulomatous inflammation were previously diagnosed, treated or currently undergoing treatment in the ETMS registry. As a result of the assessment of remaining 453 cases, 46 patients (10.1\%) were newly diagnosed with TB. Newly diagnosed TB patients were reported, and their treatment started.

Conclusion: As a result, active surveillance method conducted in pathology laboratories are used to detect unknown or late reported TB cases and allows to start treatment without further delay.

Key words: tuberculosis, granulomatous inflammation, active surveillance

Address for correspondence: O. Terzi, Ondokuz Mayis University, Faculty of Medicine, Department of Public Health, 55200-Atakum/Samsun, Turkey. E-mail: ozlem.terzi@omu.edu.tr

https://doi.org/10.21101/cejph.a6124

\section{INTRODUCTION}

Tuberculosis (TB) remains an important public health problem. According to a World Health Organization (WHO) report, the TB incidence rate is 28 (per 100,000 population) in the European Region and 17 (per 100,000 population) in Turkey. Turkey is located in the WHO European Region and is a transition zone between Europe and Asia (1).

Early detection and case notification, evaluation of contacts, detection of secondary cases, and appropriate chemoprophylaxis are necessary for the worldwide control of TB (2). TB notification is defined as the process of reporting identified TB cases to the relevant health authorities, which then notify the WHO via national TB programmes (NTPs) or their equivalents (3). The systematic assembly, classification, and analysis of the data and their distribution to the authorities who need it to take measures, is called surveillance. Surveillance can be accomplished with various methods. The passive surveillance method is the expectation that reports about the disease will come from institutions through standard forms. Regular data retrieval by various methods, such as on-site visits, telephone, or mail, is referred to as active surveillance, where a surveillance officer can search for cases (4).

Some national TB surveillance systems are inadequate in terms of patient detection, despite improvements in TB notification systems with advancing technology (5-7). In 2019, an estimated 2.9 million people of the 10 million people who fell ill with TB were not diagnosed or reported to the WHO (1). To be able to determine the cases that are not diagnosed, it is necessary to address new case detection, diagnosis errors, causes of delay in diagnosis and notification, and difficulties in the information process $(1,8)$. It can be assumed that some of the missing cases are associated with extrapulmonary TB (EPTB). Diagnosing EPTB is difficult compared to diagnosing pulmonary TB (PTB) due to the variety of symptoms that occur, the low level of suspicion among clinicians, and the difficulty of obtaining sufficient samples for verification (9). According to the WHO, in 2019 the highest incidence rate of EPTB was in the Eastern Mediterranean region (24\%), followed by South-East Asia (19\%), and Europe; the lowest was in the Western Pacific region (8\%) (1). In Turkey, $35 \%$ of 11,786 TB cases reported were EPTB (10). 
Gold standard methods to diagnose TB are clinical evaluation, sputum examination, and positive mycobacterial culture (8). For TB surveillance, various secondary data sources, such as laboratory diaries, death certificates, hospital discharge records, and pharmacy records, are used in addition to primary sources, such as direct clinical diagnosis notifications (ICD-10 codes) from hospitals or dispensaries $(11,12)$. One of the deficiencies of TB notification is the lack of investigation from different sources. Very limited information has been obtained about TB surveillance in pathology laboratories (12), although they assess acid-fast bacilli (AFB) or mycobacterial culture results $(2,13)$. Although pathological diagnosis of TB is determined by chronic granulomatous inflammation, caseous necrosis is a specific pathognomonic sign of TB and therefore such histopathological changes could serve as a secondary source of information for the active surveillance system $(14,15)$.

Reporting TB cases is mandatory in Turkey. All people diagnosed with $\mathrm{TB}$ at private and public hospitals must report to a Tuberculosis Control Dispensary (TCD) to receive TB drugs and for contact examination. TCDs follow the guidelines of the Stop TB Strategy and the International Standards for Tuberculosis Care adopted by the WHO. The surveillance system is based on TB cases. Confirmed TB cases and contact examinations are carefully investigated by TCDs. The reporting of TB cases to TCDs is undertaken by local health offices by filling in and sending standard forms online to the Ministry of Health. TCD officers analyze and process the data and send it back to peripheral offices for their records. These records are collected annually by the National TB Surveillance Research Organization (16). Data regularly collected from dispensaries using Turkey's National TB Surveillance Network has been reported to the WHO since 2005. In addition, TB active surveillance has been introduced at the national level to detect and report diagnosed cases since 2015 Reported information includes the day the patient's TB treatment started in the clinic; the day when a case with granulomatous inflammation is detected by a pathology laboratory; and the day when any of the TB drugs from the hospital pharmacy are given to the patient (patient notification is provided) (17-19). The aim of this study was to investigate suspicious cases detected via the active surveillance method in pathology laboratories and to evaluate the effectiveness of this method in terms of finding new TB cases.

\section{MATERIALS AND METHODS}

This cross-sectional study was conducted in Samsun in the period 2012-2017. The legal permits required for the study were acquired previously. Samsun has a population of over 1 million and is located in the Central Black Sea Region in the north of Turkey. Of the 81 provinces in Turkey, Samsun ranks 33rd on the socioeconomic development index. According to Ministry of Health data, TB case notification rates in Samsun between the years 2012 and 2017 range from 23 to 16.7 per 100,000 $(288,261$, 224, 222, 217, 207 cases in 2012-2017, respectively).

During the data collection process, all public and private pathology laboratories (nine units) in Samsun were visited by the provincial TB coordinator. Monthly visits have occurred regularly since January 2012. All patient reports including a finding of granulomatous inflammation (such as necrotizing granulomatous, caseous granulomatous, non-necrotizing granulomatous, largecell granulomatous, etc.) were collected from these laboratories. These histopathological changes are considered typical for TB (15, 20,21). Duplicate data were removed, and a total of 707 patients remained. Pathology samples were obtained from lymph gland aspiration material, an intrathoracic mass taken during a surgical procedure, incisional or excisional biopsy, lung parenchyma taken by wedge biopsy, cavity wall tissue, pleural tissue, or other kinds of tissue.

During the patient evaluation process, patient lists of the pathology reports were compared with the records of the Electronic TB Management System (ETMS). Patients who were on a dispensary list (patients who were still undergoing treatment or who were previously treated) were separated. All those who were not registered at any dispensary and whose place of residence was in Samsun were invited to the closest dispensary for further investigation.

Following are the procedures in the dispensary invitation:

- The patients were informed, and their approvals were taken.

- A detailed anamnesis was taken, and physical examination was performed.

- Chest graph (X-ray) was obtained for the investigation of fibrotic lesions suggestive of tuberculous sequelae on patients.

- Tuberculin skin test (TST) was performed and the result was evaluated by Mantoux method.

- At least 2 sputum specimens were taken. All specimens were transported to one TB reference laboratory within 72 hours of collection for acid-fast bacilli (AFB) smear microscopy and mycobacterial culture.

- Sputum specimens were directly stained with Ehrlich-ZiehlNeelsen or Kinyoun dyeing methods and examined microscopically.

- Some patients were validated with culture. Solid media (Lowenstein-Jensen (LJ)) or liquid media (broth-based BACTEC MGIT 960) were used for mycobacterial culture (Fig. 1).

Some of the patients were referred to the Chest Diseases Hospital (e.g., cases with severe or frequent hemoptysis, diabetes, chronic kidney or chronic liver disease, drug allergy and druginduced hepatitis, etc.). Beginning in January 2012, each patient was classified as a TB patient or not a TB patient 3-6 weeks at the latest after a dispensary or hospital examination. Necessary treatment was provided to TB patients free of charge by dispensaries as a part of the National TB Control Programme of Turkey. If the disease was determined not to be $\mathrm{TB}$, the patient was allowed to continue his/her current treatment or was referred to the relevant specialist or his/her primary physician for further investigation and diagnosis. In an evaluation of residents living elsewhere during the investigation, the TB coordinators of the relevant provinces were informed, and results were included in the study.

SPSS was used for the statistical analysis of the data obtained in the study. Patients' ages were presented as median and range because the data were not normally distributed. Frequencies were expressed as numbers and percentages. A Chi-square test was used to compare frequency data. The statistical significance level was set at $\mathrm{p}<0.05$.

The study was conducted in accordance with the Declaration of Helsinki, and the protocol was approved by the Ethics Committee of Ondokuz Mayss University, Samsun, Turkey (OMU KAEK 2018/96). 


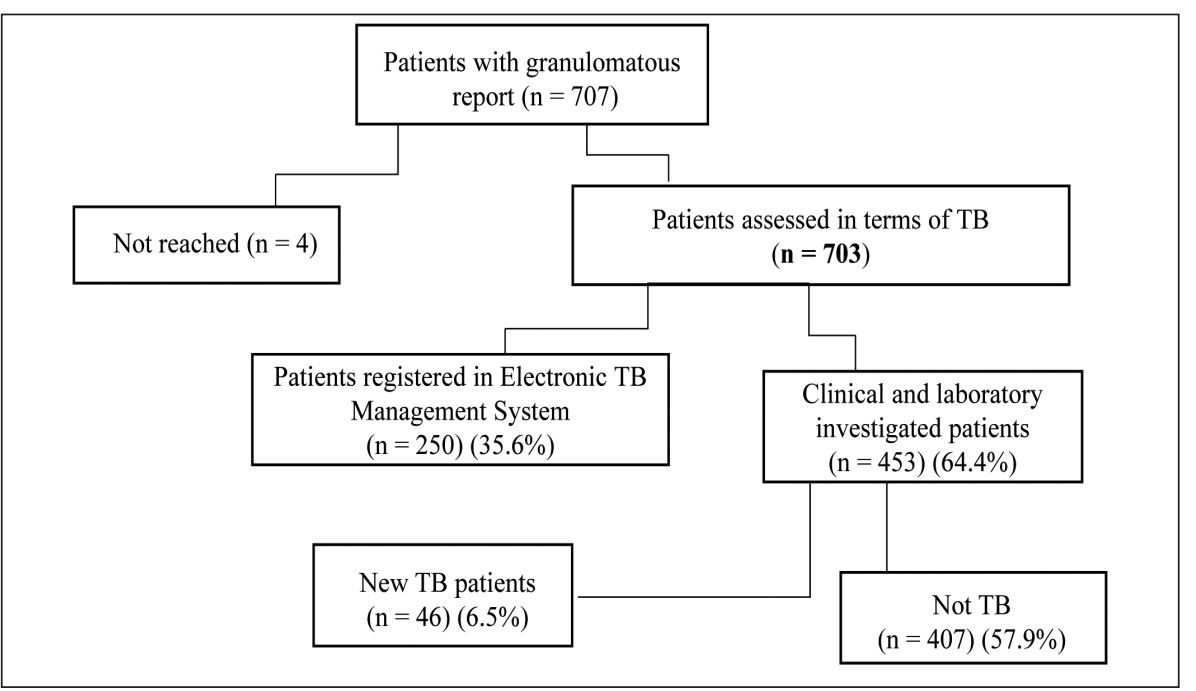

Fig. 1. Examination of TB obtained from active surveillance.

\section{RESULTS}

In the pathology report, $4(0.5 \%)$ out of 707 individuals described as being granulomatous could not be reached, and the study was completed with 703 patients. The median age of the 703 patients was 49 years (range: $2-89$ ), and $68.3 \%$ were females. The median age of the female patients was 48 (range: 2-89) years, and the median age of the male patients was 57 (range: 4-78) years. However, the difference was not statistically significant $(\mathrm{p}>0.05)$. When compared to the ETMS registry list, $250(35.6 \%)$ out of 703 people were found to be a TB-treated (formerly treated or currently treated) patients. As a result of the dispensary and hospital examinations of those not found in the ETMS registry, $407(57.9 \%)$ patients were not TB patients and $46(6.5 \%)$ were TB patients (new patients as a result of active surveillance research). Thus, with the active surveillance system, 46 (10.1\%) of the 453 patients who were pathologically reported as having granulomatous disease were TB patients (new TB). The number of new patients diagnosed from the pathology laboratories per year was calculated as 7.6 (46 patients/6 years). According to year, the ratio of patients diagnosed by means of active surveillance to total TB patients was calculated as $1.7 \%(5 / 288)$ for $2012,1.7 \%(5 / 261)$ for $2013,2.2 \%(5 / 224)$ for $2014,4.0 \%(9 / 222)$ for $2015,4.6 \%$ (10/217) for 2016, and 5.7\% (12/207) for 2017.

The median age of newly diagnosed patients was 62 (range: 19-85) years and was statistically significantly higher than that registered in the ETMS and for the non-TB groups $(\mathrm{p}<0.001)$
(Table 1). Of the new patients, $71.7 \%$ were females. Forty-six patients were referred to the dispensaries closest to their address for their TB treatment; of these, 2 were not treated due to additional diseases and senility. Comparisons of patients diagnosed through the active surveillance system, the status of TB diagnosis, and some demographic characteristics are presented in Table 1.

When the pathology reports of the patients are classified according to subtypes of granulomatous inflammation, 28 (12\%) of 233 patients with proven granulomatous disease were diagnosed with TB through active surveillance. Of these, 184 (79\%) were registered patients of a dispensary. The incidence of new diagnoses in other granulomatous patients is $5 \%$ (Table 2).

When evaluated according to TB localization, 206 (83.1\%) of the patients with EPTB were registered at a dispensary, while 42 $(16.9 \%)$ were newly diagnosed patients. There was no statistically significant difference between registered and new patient groups according to TB localization (Chi-square value: 1.6, $\mathrm{p}>0.05$ ) (Table 3 ). On the other hand, only three patients had culture positivity. Two of these patients were pulmonary TB and one was EPTB.

\section{DISCUSSION}

In our study, in 2012-2017 453 patients with granulomatous inflammation were screened by clinical methods and 46 cases $(10.1 \%)$ were reported to official authorities after being diagnosed with TB, and treatment was then started. The TB incidence rate in Turkey decreased from 17.9 in 2012 to 13.7 in 2017 (Fig. 2). In

Table 1. Demographic characteristics of data investigated by active surveillance study according to status of TB diagnosis ( $N=703)$

\begin{tabular}{|l|c|c|c|c|}
\hline & Registered in ETMS & New TB patients & No TB & p-value \\
\hline $\begin{array}{l}\text { Gender } \\
\text { Female n (\%) }\end{array}$ & $173(69.2)$ & $33(71.7)$ & $274(67.5)$ & $>0.05$ \\
\hline $\begin{array}{l}\text { Age/year } \\
\text { Median (min-max) }\end{array}$ & $49(2-89)$ & $62(19-85)$ & $47(2-87)$ & $<0.001^{*}$ \\
\hline Total n (\%) & $250(35.6)$ & $46(6.5)$ & $407(57.9)$ & \\
\hline
\end{tabular}

ETMS - electronic TB management system

${ }^{*}$ Kruskal-Wallis test and Bonferonni corrected Mann-Whitney U test 
Table 2. Distribution of patients' pathology reports according to TB status ( $N=703)$

\begin{tabular}{|l|c|c|c|}
\hline & $\begin{array}{c}\text { Registered in ETMS } \\
\mathbf{n}(\%)\end{array}$ & $\begin{array}{c}\text { New TB patients } \\
\mathbf{n}(\%)\end{array}$ & $\begin{array}{c}\text { No TB } \\
\mathbf{n}(\%)\end{array}$ \\
\hline Caseous granulomatous $(\mathrm{n}=233)$ & $184(79.0)$ & $28(12.0)$ & $21(9.0)$ \\
\hline Non-caseous granuloma $(\mathrm{n}=128)$ & $10(7.8)$ & $1(0.8)$ & $117(91.4)$ \\
\hline Other $(\mathrm{n}=342)$ & $56(16.4)$ & $17(5.0)$ & $269(78.6)$ \\
\hline Total $(\mathrm{N}=703)$ & $250(35.6)$ & $46(6.5)$ & $407(57.9)$ \\
\hline
\end{tabular}

${ }^{*}$ Other: necrotizing granulomatous, non-necrotizing granulomatous and granulomatous

ETMS - electronic TB management system, Pearson's Chi-squared - Chi²: 351.0; $p<0.001$

our study region, $\mathrm{TB}$ case notification rates decreased in the same years from 23 to 16.7 per 100,000 people. We think the incidence of undetected cases of TB increased during the study period, even though the absolute number of examined patients decreased. This may be due to the increased awareness of clinicians and pathology laboratories and the accessibility of difficult-to-diagnose cases.

In our study, we also determined that the median age of cases with granulomatous inflammation was 49 , and two thirds were women. In a study by Mukhopadhyay et al. (15), the authors examined 500 cases of pulmonary granuloma from seven different countries, including Turkey. The median age of the patients was 51, similar to our study. However, Mukhopadhyay et al. found a different gender distribution ( 1.1 male/1 female) than our study (15). This may have been caused by a difference in the frequency of the disease, which is accompanied by granulomatous inflammation in different geographies, or by a difference in the mechanisms or processes of inflammation according to gender. The gender effect is known to have a strong regulatory effect on possible confounding factors (22). The main causes of this difference can be revealed by different epidemiological study designs related to age and gender.

A finding of caseification is pathognomonic for $\mathrm{TB}$, and it is important for clinicians to be informed by pathologists about suspected TB. However, there are inadequacies about notifications of pathologically diagnosed TB cases $(17,23,24)$. A review of the clinical literature showed that $38-49 \%$ of patients whose cases were not reported by clinicians when TB was diagnosed could be detected from pathology reports with positive histopathologic features or from anti-TB drug prescriptions (25). In our study, 28 patients with caseous granuloma were diagnosed with $\mathrm{TB}$ faster as a result of active surveillance, and dispensaries started treatment quickly. This may be due to delays or disruptions in the patient notification process. Nonetheless, it can be considered that the pathologists have never reported, or the clinicians or surgeons have not adequately followed the pathology reports. Sometimes, a communication gap between clinicians and pathologists occurs. They may not be able to communicate with each other about patient reports due to reasons such as lack of medical infrastructure, workload, timelessness, or lack of staff. Detailed information about the properties of granulomas in the pathology report will help clinicians. The localization of granulomas, formation, necrosis, type of necrosis, distribution of inflammatory cell population around the granuloma, and the presence of co-vasculitis will guide the differential diagnosis. Again, in our study, we determined that $5 \%$ of patients with necrotizing or non-necrotizing granulomatous inflammation had TB through active surveillance. Although it is not a common cause for other granulomatous formations in which necrotizing or non-necrotizing granulomas are present, TB should necessarily be kept in mind in making the differential diagnosis (15).

In order to identify delays or insufficient notifications in TB notification, it is necessary to accurately evaluate existing data sources and to ensure information flow from different sources. The WHO recommends countries review and improve their TB surveillance systems every 10 years. For this purpose, it has published a checklist that allows countries to review their own systems $(26,27)$. According to a study conducted on evaluating the surveillance systems, most low-incidence countries $(<100 \mathrm{~TB}$

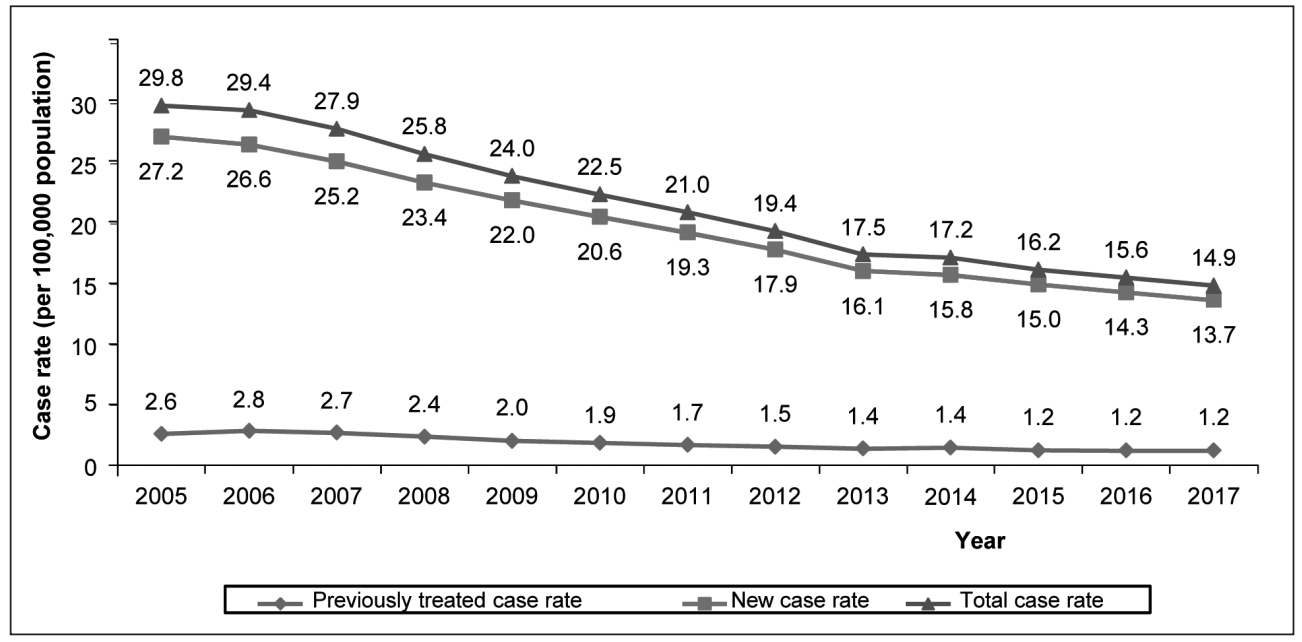

Fig. 2. TB case notification rate in Turkey, 2005-2017 (10). 
cases per million population), have individual case-based electronic TB surveillance. However, only $61 \%$ of European countries perform regular supervision and only $39 \%$ have a monitoring and evaluation plan. Countries that regularly monitor and evaluate TB surveillance include Central and Eastern European countries such as Hungary, Romania, and Slovakia (28).

In terms of early notification of TB cases, it is stated that the notification rates of health service providers (insurance companies, etc.) are higher than those of laboratories, hospital staff, or other entities $(2,17)$. A study conducted in India found that lost TB cases were observed to be higher there because of the lack of reporting from private health institutions (29). In a study conducted in Brazil, it was shown that some parts of the country lacked timeliness and acceptability for TB notifications (6). In our study, dispensary employees were able to extract data by using the active surveillance method from pathology laboratories as a different data source. It is also believed that pathologists helped increase awareness of TB by visiting both state and private sector laboratories. Communication and cooperation between all health providers and services, particularly clinicians and public health workers, are important to establish a high-quality system of TB surveillance (30).

One of the important findings of our study is related to EPTB. According to the data of TB cases in 2017 in Turkey, it has been reported that $33.9 \%$ of all TB cases had EP involvement (10). In the same report, the rate of EPTB in Samsun was $36.7 \%$ (10). In our study, the rate of EPTB was higher. In fact, the diagnosis of EPTB is often delayed or even missed due to insidious clinical presentation and the poor performance of diagnostic tests. Cultures, the classic gold standard for TB testing, suffer from increased technical and logistical constraints in EPTB cases (31). Solovic et al. (9) evaluated the difficulties of diagnosing EPTB in the European Union/European Economic Area countries. The countries that reported challenges in the diagnosis of EPTB reported that EPTB is often not considered because it is rare and most medical professionals do not have experience in diagnosing it. Diagnosis of EPTB poses challenges due to the diversity of symptoms, the low level of suspicion among clinicians, and the difficulty in obtaining an adequate sample for confirmation. For more timely and adequate diagnosis, awareness of EPTB should be increased among non-pulmonary physicians, and guidelines for diagnosis and treatment of EPTB should be more widely available (9).

In a study conducted in the United States, it was reported that even though EPTB patients were definitely diagnosed, their notifications were made later than those of pulmonary TB patients. The study stated that these delays may be caused by the fact that notifications were not done promptly because EPTB does not carry the risk of contagion (2). It was found that 42 of the 46 recently diagnosed TB patients were EPTB cases. For this reason, we think that EPTB cases, which clinicians do not think TB primarily but with granulomatous inflammation as a pathologic finding, can be determined by active surveillance by pathology laboratories and thus confirmed and treated earlier.

In our study, it was determined that the majority of newly diagnosed EPTB cases were females and of older age. It is generally stated that women are more at risk for EPTB, and the role of endocrine factors in the body, cellular immunity, iron metabolism, socioeconomic barriers, and stigma can all be associated with gender-specific differences (32).

\section{CONCLUSION}

To better understand TB outbreaks in communities and to end these outbreaks and stop TB on a global level, it is emphasized that data quality should be increased and reporting systems should be modernized at all levels (33). It is only with a strong surveillance system and improved reporting that the number of lost TB cases can be reduced (34). The cost of active surveillance was not considered as a limitation of our study. However, it will be necessary to determine cost efficiency through further research. In conclusion, the establishment of an active notification system at the national level rather than passive methods by pathology laboratories will contribute to the initiation of early treatment by detecting unknown or late reported cases. We believe that active patient reporting by pathology laboratories in countries such as Turkey that have national TB notification systems is an enforceable policy but one that depends on existing infrastructure and the competency of various personnel.

\section{Acknowledgements}

We would like to thank to Samsun Provincial Tuberculosis Dispensary and Chest Hospital staff for their contribution.

\section{Conflict of Interests}

None declared

\section{REFERENCES}

1. World Health Organization. Global tuberculosis report 2019 [Internet]. Geneva: WHO; 2019 [cited 2021 Jan 10]. Available from: https://apps. who.int/iris/bitstream/handle/10665/329368/9789241565714-eng.pdf.

2. Curtis AB, McCray E, McKenna M, Onorato IM. Completeness and timeliness of tuberculosis case reporting: a multistate study. Am J Prev Med. 2001;20(2):108-12.

3. Tollefson D, Ngari F, Mwakala M, Gethi D, Kipruto H, Cain K, et al. Under-reporting of sputum smear-positive tuberculosis cases in Kenya. Int J Tuberc Lung Dis. 2016;20(10):1334-41.

4. World Health Organization. WHO recommended surveillance standards. 2nd ed. [Internet]. Geneva: WHO; 1999 [cited 2021 Jan 10]. Available from: http://apps.who.int/iris/handle/10665/65517.

5. Ghosh S, Moonan PK, Cowan L, Grant J, Kammerer S, Navin TR. Tuberculosis genotyping information management system: enhancing tuberculosis surveillance in the United States. Infect Genet Evol. 2012;12(4):782-8.

6. Silva GDMD, Bartholomay P, Cruz OG, Garcia LP. Evaluation of data quality, timeliness and acceptability of the tuberculosis surveillance system in Brazil's micro-regions. Cien Saude Colet. 2017 Oct;22(10):3307-19.

7. Migliori GB, Spanevello A, Ballardini L, Neri M, Gambarini C, Moro ML, et al; Varese Tuberculosis Study Group. Validation of the surveillance system for new cases of tuberculosis in a province of northern Italy. Eur Respir J. 1995 Aug;8(8):1252-8.

8. Romero ROG, Ribeiro CMC, Sá LD, Villa TCS, Nogueira J de A. Underreporting of tuberculosis cases from death surveillance. Rev Eletr Enferm [Internet]. 2016 Jun 30 [cited 2021 Jan 25];18. Available from: https:// revistas.ufg.br/fen/article/view/37249.

9. Solovic I, Jonsson J, Korzeniewska-Koseła M, Chiotan DI, Pace-Asciak A, Slump E, et al. Challenges in diagnosing extrapulmonary tuberculosis in the European Union, 2011. Euro Surveill. 2013 Mar 21;18(12). pii: 20432.

10. Report on tuberculosis in Turkey, 2019 [Internet]. Ankara: HSGM; 2020 [cited 2021 Jan 10]. Available from: https://hsgm.saglik.gov.tr/ tr/tuberkuloz-haberler/turkiye-de-verem-savas-2019-raporu.html. (In Turkish.)

11. Siddiqi K, Lambert ML, Walley J. Clinical diagnosis of smear-negative pulmonary tuberculosis in low-income countries: the current evidence. Lancet Infect Dis. 2003;3(5):288-96. 
12. Driver CR, Braden CR, Nieves RL, Navarro AM, Rullan JV, Valway SE, et al. Completeness of tuberculosis case reporting, San Juan and Caguas Regions, Puerto Rico, 1992. Public Health Rep. 1996;111(2):157-61.

13. San Gabriel P, Saiman L, Kaye K, Silin M, Onorato I, Schulte J. Completeness of pediatric TB reporting in New York City. Public Health Rep. 2003;118(2):144-53.

14. Lai S, Wangteeraprasert T, Sermkaew T, Nararak O, Binsard S, Phanawadee M, et al. Evaluation of three main tuberculosis case reporting systems in Satun Province, Thailand, 2011. Outbreak Surveill Investig Rep [Internet]. 2014 [cited 2021 Jan 25];7(3):16-23. Available from: http://www.osirjournal.net/index.php/osir/article/view/37.

15. Mukhopadhyay S, Farver CF, Vaszar LT, Dempsey OJ, Popper HH, Mani $\mathrm{H}$, et al. Causes of pulmonary granulomas: a retrospective study of 500 cases from seven countries. J Clin Pathol. 2012;65(1):51-7.

16. McAdam AJ, Sharpe AH. Infectious diseases. In: Kumar V, Abbas AK, Aster JC, editors. Robbins and Cotran pathologic basis of disease. 9th ed. Philadelphia: Elsevier/Saunders; 2015. p. 371-6.

17. Türkkani MH, Özdemir T, Akkuş İH. Should tuberculosis reporting be done from the laboratories? J Izmir Chest Hosp. 2019;33(3):145-51. (In Turkish.)

18. Türkkanı MH, Koyuncu E, Özkara S, Güneri FD, Özdemir T. The Problems about the Management of Tuberculosis Control Program for the Patients with Vertebral Tuberculosis. Solunum Hast Derg. 2015;25 (2-3):11-6. (In Turkish.)

19. Kara F, editor. Tuberculosis active surveillance criteria. 2nd ed. [Internet]. Ankara: Ministry of Health, Turkey; 2019 [cited 2021 Jan 10]. Available from: https://hsgm.saglik.gov.tr/depo/birimler/tuberkuloz_db/haberler/ Tuberkuloz Tani Ve Tedavi Rehberi/Tuberkuloz Tani ve Tedavi Rehberi.pdf. (In Turkish.)

20. Mukhopadhyay S. Non-neoplastic pulmonary pathology: an algorithmic approach to histologic findings in the lung. Cambridge: Cambridge University Press; 2016.

21. Jain D, Ghosh S, Teixeira L, Mukhopadhyay S. Pathology of pulmonary tuberculosis and non-tuberculous mycobacterial lung disease: facts, misconceptions, and practical tips for pathologists. Semin Diagn Pathol. 2017 Nov;34(6):518-29.

22. Yone EW, Kengne AP, Moifo B, Kuaban C. Prevalence and determinants of extrapulmonary involvement in patients with pulmonary tuberculosis in a Sub-Saharan African country: a cross-sectional study. Scand J Infect Dis. 2013 Feb;45(2):104-11.

23. Mukhopadhyay S, Gal AA. Granulomatous lung disease: an approach to the differential diagnosis. Arch Pathol Lab Med. 2010;134(5):667-90.
24. Park DY, Kim JY, Choi KU, Lee JS, Lee CH, Sol MY, et al. Comparison of polymerase chain reaction with histopathologic features for diagnosis of tuberculosis in formalin-fixed, paraffin-embedded histologic specimens. Arch Pathol Lab Med. 2003;127(3):326-30.

25. Pillaye J, Clarke A. An evaluation of completeness of tuberculosis notification in the United Kingdom. BMC Public Health. 2003 Oct 6;3:31. doi: 10.1186/1471-2458-3-31.

26. World Health Organization. Global tuberculosis control: WHO report 2001. Geneva: WHO; 2001.

27. World Health Organization. WHO TB surveillance checklist [Internet]. Geneva: WHO; 2013 [cited 2021 Jan 10]. Available from: http://www. who.int/tb/meetings/accra.

28. Lönnroth K, Migliori GB, Abubakar I, D'Ambrosio L, De Vries G, Diel $\mathrm{R}$, et al. Towards tuberculosis elimination: an action framework for lowincidence countries. Eur Respir J. 2015;45(4):928-52.

29. Satpati M, Burugina Nagaraja S, Shewade HD, Aslesh PO, Samuel B, Khanna A, et al. TB notification from private health sector in Delhi, India: challenges encountered by programme personnel and private health care providers. Tuberc Res Treat. 2017;2017:6346892. doi: $10.1155 / 2017 / 6346892$.

30. Van der Werf MJ, Sotgiu G, Dara M. Closing the gap in surveillance of tuberculosis and HIV co-infection: a European perspective on the need for clinician-public health alliances. Eur Respir J. 2017 Nov 16;50(5):1701758. doi: 10.1183/13993003.01758-2017.

31. Norbis L, Alagna R, Tortoli E, Codecasa LR, Migliori GB, Cirillo DM. Challenges and perspectives in the diagnosis of extrapulmonary tuberculosis. Expert Rev Anti Infect Ther. 2014;12(5):633-47.

32. Kang W, Yu J, Du J, Yang S, Chen H, Liu J, et al. The epidemiology of extrapulmonary tuberculosis in China: a large-scale multi-center observational study. PLoS One. 2020;15(8):e0237753. doi: 10.1371/journal. pone. 0237753

33. Theron G, Jenkins HE, Cobelens F, Abubakar I, Khan AJ, Cohen T, et al. Data for action: collection and use of local data to end tuberculosis. Lancet. 2015;386(10010):2324-33.

34. Zumla A, George A, Sharma V, Herbert RH, Oxley A, Oliver M. The WHO 2014 global tuberculosis report - further to go. Lancet Glob Health. 2015 Jan;3(1):e10-2.

Received February 4, 2020

Accepted in revised form January 25, 2021 